\title{
Sharp Interface Level Set Method Based Simulation and Energy Budget Analysis of Falling Droplet Breakup Modes
}

\author{
Yashraj Bhosale, Javed Shaikh, Atul Sharma \\ Indian Institute of Technology Bombay \\ Powai, Mumbai, India \\ yashraj@iitb.ac.in; javed.shaikh@iitb.ac.in; atulsharma@iitb.ac.in
}

\section{Extended Abstract}

Droplet dynamics is of great importance from engineering and a scientific point of view. Droplet breakup mechanisms are seen in many different industrial applications like paint sprays, ink jet printers and liquid fuel atomization in combustion as well as natural phenomenon like raindrops. Breakup types and their variation with surface tension, viscosity and density ratio for an individual drop falling under gravity have been studied by Han et al. [1] and Fakhari et al. [2] and correlation with drag coefficients studied by Fakhari et al. [3]. Han et al. [1] studied the transition between different break up modes by varying density ratios, viscosity ratios, surface tension and the effects have been summarised in regime maps while Fakhari et al [3] presented variation of drag coefficient along with these transitions. The differences between drop fall and bubble rise dynamics have been studied by Tripathi et al. [4] for different non dimensional numbers.

In this study, the objective is to simulate the breakup of liquid drops under the action of gravity, using a numerical method - ghost fluid method based sharp interface level set method (SI-LSM) - which can accommodate large deformation of the falling drops. Furthermore, the objective is to capture various breakup modes, with the variation of fluid properties; and analyse the modes by the energy budget plots. Finally, the objective is to demonstrate direct correspondence between the breakup modes and its temporal energy evolution.

The governing equations (continuity, momentum and level set) for the axisymmetric domain are solved numerically on a uniform dual grid using SI-LSM. An axisymmetric domain-size of $3 \times 12$ is chosen for the simulations (grid size $250 \times 1000$ ). The left boundary is set as symmetric wall and outflow boundary condition is applied on the other boundaries. The fundamental parameters varied are the density ratio (ratio of drop density to the outside fluid density - 1.15 and 10), the Ohnesorge number (ratio of viscous forces to inertial and surface tension forces -0.05 to 0.250 ) and Eotvos number (ratio and surface tension force and body forces - 12 to 144). Simultaneously, the different energy components which include potential energy, surface energy, kinetic energy and viscous dissipation are calculated and their temporal variation and contribution leading to the breakup of the drop are studied.

Excellent quantitative validation is found, for aspect ratio and velocity profile, for two particular cases of density ratio of a falling droplet. The mass error in the simulation up to the breakup has been observed to be less than 5\%. Acceptable qualitative validation has been obtained for the developing falling drop shape with time for all the cases with the work of Han et al. [1]. With variation of fluid properties different secondary break up modes like oblate ellipsoid, oscillating, shear, back ward bag and forward bag types are observed. A characteristic type of energy budget plot is found for each type of breakup mode for the different non dimensional numbers considered in this study. With increasing Eotvos number, for low density ratio, transition of breakup type from oblate ellipsoid via forward bag to backward bag type breakup is observed which is revealed by the energy plots as well. For high density ratio, increase in Ohnesorge number leads to increase in viscous dissipation, which causes transition from shear type breakup to backward bag type breakup mode. Increase in viscosity ratio also leads to increased viscous dissipation within the drop causing rapid decrease in kinetic energy and results in transition of shear type breakup to forward bag type breakup mode. The transition in break up types observed in the simulations is also demonstrated by the energy budget plots.

Keywords: Sharp Interface level set method, droplet breakup modes, energy budget 


\section{References}

[1] J. Han and G. Tryggvason, "Secondary breakup of axisymmetric liquid drops. I. Acceleration by a constant body force," Physics of Fluids, vol. 11, no. 12, pp. 3650-3667, 1999.

[2] A. Fakhari, M. H. Rahimian, "Simulation of falling droplet by the lattice Boltzmann method," Commun Nonlinear Sci Numer Simulat, vol. 14, no. 7, pp. 3046-3055, 2009.

[3] A. Fakhari and M. H. Rahimian, "Investigation of deformation and breakup of a falling droplet using a multiplerelaxation-time lattice Boltzmann method," Computers \& Fluids, vol. 40, no. 1, pp. 156-171, 2011.

[4] M. K. Tripathi, K. C. Sahu, R. Govindarajan, "Why a falling drop does not in general behave like a rising bubble," Scientific Reports, vol. 4, pp. 4771-4771, 2014. 